International Mathematical Forum, 2, 2007, no. 5, 243 - 249

\title{
The Theory of Multidimensional Differential Equations in Lie Group and Conditions for Their Exact Solvability
}

\author{
Mehmet Şahin \\ Gaziantep University, Faculty of Arts and Sciences \\ Department of Mathematics, Gaziantep, Turkey
}

\section{B. Ragimov}

Baku State University, Baku, Azerbaijan

\begin{abstract}
In this study, we investigate multidimensional differential equation with special coefficient. We built a new theorem for necessary and sufficient conditions for solvability of these differential equations. The proof of this theorem gives, n-dimensional vector spaces, $R_{x}^{n}$ which transform Lie algebra. As a result of this transformation, we find new conditions for exact integrable of multidimensional differential equations.
\end{abstract}

Mathematics Subject Classification: 22E60, 17A36, 81U15

Keywords: Lie Group, Monomorph Operator, Exact Solvability

\section{INTRODUCTION}

Let $R_{x}^{n}$ be an n-dimensional space of vectors and $\mathrm{E}$ be an arbitrary complex Banach space, $L\left(R_{x}^{n} ; E\right)$ be space of operators space which acts from $R_{x}^{n}$ to $\mathrm{E}$ and $C\left(R_{x}^{n} ; L(E ; E)\right)$ be space of strong continuous operators acting from $R_{x}^{n}$ to Endomorphism algebra $L(E ; E)$ of Banach space E. The spectral theory of this kind of operator family has been investigated in [1]

2. Nonhomogeneous Multidimensional Differential Equation

Consider the multidimensional differential equation below

$$
u^{\prime}(x) h=A(Q(x) h) u(x)+f(x) h
$$

The initial condition is;

$$
u\left(x_{0}\right)=u_{0},\left(x_{0} \in S, u_{0} \in E\right)
$$


Here, the meaning of the differentiation $u^{\prime}(x) \in L\left(R_{x}^{n} ; E\right)$ is Freshe. In addition, $x \in S, h \in R_{x}^{n} ; u(x) \in E, Q(x) \in G L(n ; R)$ and $\mathrm{S}$ is zero neighborhood in $R_{x}^{n}$. Let $\mathrm{Q}$ be an element of $C^{\infty}(S)$ and $Q(0)=I$ (identity matrices), A be an element of $C\left(R_{x}^{n} ; L(E ; E)\right)$ at that $(A h \neq 0)$ on $h \neq 0$ and the operator function $f$ which acts from $R_{x}^{n}$ to $L\left(R_{x}^{n} ; E\right)$ be differentiable and continuous is the neighborhood of $S \subset R_{x}^{n}$.

Definition 1. If for an arbitrary point $\left(x_{0}, u_{0}\right)\left(\right.$ i.e. $\left.x_{0} \in S, u_{0} \in E\right)$, (2.1) is defined around $x_{0}$ and satisfies the initial conditions $u\left(x_{0}\right)=u_{0}$, then it has a continuous differentiable solution and is called solvable or exact integrable.

Definition 2. Let's donate $L(E ; E)$ as F. Following Shilov [2], the mapping $A: R_{x}^{n} \rightarrow F$ will be called morphism if the following relations hold true

1. $A\left(h_{1}+h_{2}\right)=A h_{1}+A h_{2}$, for any arbitrary $h_{1}, h_{2} \in R_{x}^{n}$,

2. $A(\alpha h)=\alpha A h$, for any arbitrary $h \in R_{x}^{n}, \alpha \in C$.

If the morphism $\mathrm{A}$ maps the space $R_{x}^{n}$ onto the whole spaces $\mathrm{F}$, it is called epimorphism. If the morphism A maps the space $R_{x}^{n}$ at least not onto the whole spaces $\mathrm{F}$ but one-to-one (i.e. from $h_{1} \neq h_{2}$, it follows that $A\left(h_{1}\right) \neq A\left(h_{2}\right)$, it is called monomorphism.

Definition 3. Following N.Bourbaki [3], if the below multiplication

$$
(A h, B k) \Longrightarrow[A h, B k], \forall k, h \in R_{x}^{n}
$$

holds the following three conditions in $F$ algebra, then $F$ is called Lie algebra.

1. $[A h, A h]=0, h \in R_{x}^{n}, A h \in F$;

2. $[A h, B k]=A h B k-B k A h=-[B k A h-A h B k]=-[B k, A h], \forall A h, B k \in$ $F, h, k \in R_{x}^{n}$

3. $[A h,[B k, C \xi]]+[B k,[C \xi, A h]]+[C \xi,[A h, B k]]=0, \forall h, k, \xi \in R_{x}^{n}$.

Here $A h, B k, C \xi$ are in $\mathrm{F}$ and the symbol $[A h, B k]$ is the commutator of operators $A h, B k$ (i.e. $\left.[A h, B k]=(A h B k-B k A h), \forall h, k \in R_{x}^{n}\right)$.

Theorem 1. The necessary and sufficient conditions for the exact solvability of (2.1) are;

1. In $R_{x}^{n}$ vector spaces there is a Lie product defined as;

$$
\gamma(h, k)=[h, k]=Q^{\prime}(x)(h, k)-Q^{\prime}(x)(k, h), \forall h, k \in R_{x}^{n}
$$

2. According to the above product the following conditions hold

a): $A[h, k]=[A h, A k]$;

b): $Q^{\prime}(x)(h, k)-Q^{\prime}(x)(k, h)=[Q(x) h, Q(x) k]$;

c): $\Lambda_{h k}\{A h \varphi(x) k\}+\Lambda_{h k}\left\{\varphi^{\prime}(x) h k\right\}=0, \forall h, k \in R_{x}^{n}$

Here the symbol [.,.] is the commutator of operators Ah and Ak which are in $\mathrm{F}$ and $\Lambda_{h k}$ is the co-symmetric operator.

Proof. Assume that (2.1) is exact solvable. This shows that for an arbitrary point $\left(x_{0}, u_{0}\right), x_{0} \in S, u_{0} \in E$ this equation which is defined around $x_{0}$ and 
satisfy the initial condition $u\left(x_{0}\right)=u_{0}$ has continuous differentiable solution. The function $u(x)$ which holds the above properties is the solution for (2.1). If we put this solution into (2.1) then we obtain the following equation

$$
u^{\prime}(x) h=A(Q(x) h) u(x)+f(x) h
$$

If we differentiate $(2.3)$, then we obtain

$$
\begin{gathered}
u^{\prime \prime}(x) h k=A\left(Q^{\prime}(x)(h, k)\right) u(x)+A(Q(x) h) u^{\prime}(x) k+f^{\prime}(x) h k \\
=A\left(Q^{\prime}(x)(h, k)\right) u(x)+A(Q(x) h)[A(Q(x) k) u(x)+f(x) k]+f^{\prime}(x) h k
\end{gathered}
$$

$$
=A\left(Q^{\prime}(x)(h, k)\right) u(x)+A(Q(x) h) A(Q(x) k) u(x)+A(Q(x) h) f(x) k+f^{\prime}(x) h k \text {. }
$$

Since the second order differential solution is symmetric with respect to $h$ and $\mathrm{k}$, we get from (2.4),

$$
A\left(Q^{\prime}(x)(h, k)\right) u(x)+A(Q(x) h) A(Q(x) k) u(x)+A(Q(x) h) f(x) k+f^{\prime}(x) h k
$$

$$
A\left(Q^{\prime}(x)(k, h)\right) u(x)+A(Q(x) k) A(Q(x) h) u(x)+A(Q(x) k) f(x) h+f^{\prime}(x) k h .
$$

If we group (2.5) we get

$A\left[\left(Q^{\prime}(x)(h, k)-Q^{\prime}(x)(k, h)\right)\right] u(x)=[A(Q(x) h) A(Q(x) k)-A(Q(x) k) A(Q(x) h)] u(x)$

$$
A(Q(x) h) f(x) k-A(Q(x) k) f(x) h+f^{\prime}(x) h k-f^{\prime}(x) k h=0
$$

In (2.6) if we write $x_{0}$ instead $\mathrm{x}$ then the initial condition $u\left(x_{0}\right)=u_{0}$ holds. Here $u_{0}$ is an arbitrary vector of Banach space E. Therefore from (2.6) for any arbitrary $x_{0} \in S$ we find

$$
\begin{aligned}
A\left[\left(Q^{\prime}\left(x_{0}\right)(h, k)-Q^{\prime}\left(x_{0}\right)(k, h)\right)\right] & =\left[A\left(Q\left(x_{0}\right) h\right) A\left(Q\left(x_{0}\right) k\right)-A\left(Q\left(x_{0}\right) k\right) A\left(Q\left(x_{0}\right) h\right)\right] \\
& =\left[A\left(Q\left(x_{0}\right) h\right), A\left(Q\left(x_{0}\right) k\right)\right] .
\end{aligned}
$$

Then from this equation we find

$$
A\left[\left(Q^{\prime}\left(x_{0}\right)(h, k)-Q^{\prime}\left(x_{0}\right)(k, h)\right)\right]=\left[A\left(Q\left(x_{0}\right) h\right), A\left(Q\left(x_{0}\right) k\right)\right] .
$$

Right hand side of (2.8) is commutator of operators $A\left(Q\left(x_{0}\right) h, A\left(Q\left(x_{0}\right) k\right.\right.$ which is in F. If we choose $\mathrm{x}=0$ in $(2.8)$ then

$$
A\left(Q^{\prime}(0)(h, k)-Q^{\prime}(0)(k, h)\right)=[A h, A k] .
$$

Let's define the symbol $\gamma(h, k)$ as

$$
\gamma(h, k)=[h, k]=Q^{\prime}(0)(h, k)-Q^{\prime}(0)(k, h)
$$

In virtue of $(2.8)$ and $\mathrm{A}$ is a monomorph operator in $C\left(R_{x}^{n} ; F\right)$ then $\gamma(h, k)$ characterizes the Lie product in space of vectors $R_{x}^{n}$.

Directly 
1. $\gamma(h, h)=[h, h]=Q^{\prime}(0)(h, h)-Q^{\prime}(0)(h, h)=0$;

2. $\gamma(h, k)=Q^{\prime}(0)(h, k)-Q^{\prime}(0)(k, h)=[h, k]=-\left[Q^{\prime}(0)(k, h)-Q^{\prime}(0)(h, k)\right]=$ $-\gamma(k, h)=-[k, h]$

3. $z \in R_{x}^{n}$ holds the Jacobi equality which is below

$$
[h, \gamma(k, z)]+[k, \gamma(z, h)]+[z, \gamma(h, k)]=0
$$

Thus the space of vectors $R_{x}^{n}$ is transformed to the Lie Algebra. If we put (2.10) in (2.9) we obtain

$$
A[h, k]=[A h, A k]=A h A k-A k A h
$$

It is obvious that the operator $A \in C\left(R_{x}^{n} ; L(E ; E)\right)$ is a morphism which acts from the Lie algebra $R_{x}^{n}$ to endomorphisms $\mathrm{F}$ of Banach space E. We also know that the operator $\mathrm{A}$ is monomorph operator. Now let us use the monomorphism of $\mathrm{A}$, from (2.8) we find a commutator differential equation which depends on function $Q(x)$ :

$$
Q^{\prime}(x)(h, k)-Q^{\prime}(x)(k, h)=[Q(x) h, Q(x) k]
$$

Then if we divide both sides of (2.7) by 2 ,

$$
\frac{1}{2}[A(Q(x) h) f(x) k-A(Q(x) k) f(x) h]+\frac{1}{2}\left[f^{\prime}(x) h k-f^{\prime}(x) k h\right]=0
$$

or

$$
\Lambda_{h k}\{A(Q(x) h) f(x) k\}+\Lambda_{h k}\left\{f^{\prime}(x) h k\right\}=0, \forall h, k \in R_{x}^{n}
$$

Therefore the following conditions are satisfied in order to get an exact solution of (2.1)

$$
\begin{gathered}
A[h, k]=[A h, A k] ; \\
\Lambda_{h k}\{A(Q(x) h) f(x) k\}+\Lambda_{h k}\left\{f^{\prime}(x) h k\right\}=0 ; \\
Q^{\prime}(x)(h, k)-Q^{\prime}(x)(k, h)=[Q(x) h, Q(x) k] .
\end{gathered}
$$

Since the theorem of G. Frobenius [4] for exact solvable multidimensional differential equation, the conditions in (2.14) are also sufficient conditions for $(2.1)$.

Corollary 2. Let $Q(x)=I$, then $\forall x \in R_{x}^{n}$ (2.1) and (2.2) transform mutually to the following equations:

$$
\begin{gathered}
u^{\prime}(x) h=A h u(x)+f(x) h, \forall h \in R_{x}^{n} . \\
u\left(x_{0}\right)=u_{0}, x_{0} \in S, u_{0} \in E .
\end{gathered}
$$

The necessary and sufficient conditions for exact solvable of (2.15) and (2.16) are the following

$$
\Lambda_{h k}\{A(h) A(k)\}=0, \forall h, k \in R_{x}^{n}
$$




$$
\Lambda_{h k}\{A(h) f(x) k\}+\Lambda_{h k}\left\{f^{\prime}(x) h k\right\}=0
$$

Here, for bilinear operator $B: R_{x}^{n} \oplus R_{x}^{n} \rightarrow C\left(R_{x}^{n} ; F\right), \Lambda_{h k}$ is;

$$
\Lambda_{h k}\{B h k\}=\frac{1}{2}(B h k-B k h) .
$$

If the conditions (2.17) and (2.18) hold, then the solution of (2.15) and (2.16) follows

$$
u(x)=e^{A\left(x-x_{0}\right)} u_{0}+\int_{x_{0}}^{x} e^{A(x-\sigma)} f(\sigma) d \sigma
$$

\section{Applications}

Let $\mathrm{G}$ be $m^{2}$-dimensional Lie group and $\alpha=\left(\alpha_{11}, \ldots, \alpha_{m m}\right)$ is the collection of $m^{2}$ parameters $(\operatorname{det} \alpha \neq 0)$, characterizing the elements of this group, $E_{y}$ is the complex Banach space. Let's denote by $M\left(G ; L\left(E_{y} ; E_{y}\right)\right)$ the space of continuous operator valued maps, defined on the group $\mathrm{G}$ with the values in the algebra $L\left(E_{y} ; E_{y}\right)$ of endomorphisms of Banach space $E_{y}[6]$ - [7] - [8] - [9], with topology, given by the norm.

Suppose that the unity e of the group $G$ corresponds to the zero values of the parameters $\alpha_{11}, \ldots, \alpha_{m m}$. Let $T \in M\left(G ; L\left(E_{y} ; E_{y}\right)\right)$ since the giving of the parameters simply determines the element of the group $\mathrm{G}$ then the operator $T(g) \in M\left(G ; L\left(E_{y} ; E_{y}\right)\right), g \in G$ can be considered as the function of the parameters $\alpha_{11}, \ldots, \alpha_{m m}$, i.e. $T(g)=T\left(\alpha_{11}, \ldots, \alpha_{m m}\right)=T(\alpha)$. Consider the operator equation of the form:

$$
T(f g)=T(f) T(g),(f, g \in G)
$$

with initial condition

$$
T(e)=I,\left(e \in G ; I \in L\left(E_{y} ; E_{y}\right)\right)
$$

Where $T \in M\left(G ; L\left(E_{y} ; E_{y}\right)\right)$, and $I \in L\left(E_{y} ; E_{y}\right)$ are identical operators. Denote by $\alpha_{i j}(f), i, j=1,2, \ldots, m$ the parameters characterizing element $\mathrm{f}$ of the group G. If determining of the parameters $\alpha_{i j}(f), \alpha_{i j}(g), i, j=1,2, \ldots, m$ simply determines the element and $f, g \in G$ also determines their product $f g \in G$, and consequently, the parameters $\alpha_{i j}(f g), i, j=1,2, \ldots, m$. Let $\varphi_{t s}, t, s=1,2, \ldots, m$ are the functions of $2 m^{2}$ parameters

$\alpha_{i j}(f), a_{i j}(g): \alpha_{t s}(f g)=\varphi_{t s}\left\{\alpha_{11}(f), \ldots, \alpha_{m m}(f) ; \alpha_{11}(g), \ldots, \alpha_{m m}(g)\right\} t, s=1,2, \ldots, m$ such $\varphi_{t s}(t, s=1,2, \ldots, m)$ that are continuously differentiable functions of comultipliers. It is not difficult to see that the operator equation (3.1) - (3.2) is equivalent to the following vector equation

$$
\begin{gathered}
y(f)=T(f g) y\left(g^{-1}\right),\left(f, g, g^{-1} \in G\right) \\
y(e)=\eta\left(e \in G ; \eta \in E_{y} ; T(f) \eta=y(f)\right)
\end{gathered}
$$


The following problem is formulated by us: it is required to find the solutions $y(t) \in E_{y}, f \in G$ of the functional problems (3.3) - (3.4), analytical in the neighborhood of the unique element $f=e \in G$.

Theorem 3. Let the operator $T: G \rightarrow L\left(E_{y} ; E_{y}\right)$ has continuos private derivatives for each of the parameters $\alpha_{i j}(f), i, j=1,2, \ldots, m$ at the point $g=f^{-1} \in G$ and let the function $y(f)(f \in G)$ with continuous private derivatives $\frac{\partial y(f)}{\partial \alpha_{t s}(f)}(t, s=1,2, \ldots, m)$ is the solution of the vector equation (3.3). Then there exists the unique finite collection of linear operators $\left\{A_{11}, A_{12}, \ldots, A_{m m}\right\} \subset$ $L\left(E_{y} ; E_{y}\right)$ such that at the same time and the solution of overdetermined system of differential equations:

$$
\left(L_{i j} y\right)(f) \equiv \frac{\partial y(f)}{\partial \alpha_{i j}(f)}-\sum_{t=1}^{m} \sum_{s=1}^{m} c_{t s}^{i j}(f) A_{t s} y(f)=0
$$

The operators $L_{11}, L_{12}, \ldots, L_{m m}\left[L_{i j} \equiv \frac{\partial}{\partial \alpha_{i j}(f)}-\sum_{t=1}^{m} \sum_{s=1}^{m} c_{t s}^{i j}(f) A_{t s}\right]$ are called characteristic differential operators (CDO) of Lie, $c(e)=\left\{c_{t s}^{i j}(e)\right\}$ is mxm matrix such that $c_{t s}^{i j}(e)=\delta_{t s}^{i j}$ is Kronecker symbol. System of equations (3.5), according to Lie [10], we'll call if characteristics differential equations [GCDE] of Lie of the presentation the group G. This notion plays the important role in quantum mechanics [11]. It is true the following inverse.

Theorem 4. If SCDE of Lie (3.5) has unique analytical solution in the neighborhood of unity element $e \in G$ with initial condition (3.4), then it is also solution of the vector equation (3.3).

Definition 4. SCDE of Lie (3.5) called absolutely integrable (absolutely solvable) on the set $G_{a} \times D_{y} \subset G \times E_{y}$ for any point $(e, \eta) \in G_{0} \times D_{y}$ there exists unique solution $y(f)$ of this system, defined in some neighborhood of unity element e in $G_{a} \subset G$ which has the values in $D_{y} \subset E_{y}$ and satisfies the initial condition $y(e)=\eta$.

Theorem 5. Let $A_{t s} \in L\left(E_{y} ; E_{y}\right), t, s=1,2, \ldots, m$ are bounded, infinitesimal operators of the presentation Lie group. Suppose that the functions $\left\{G_{t s}^{i j}(f), i, j\right.$, $t, s=1,2, \ldots, m\}$ are continuously differentiable. The neighborhood of the unity element e $\in G_{0}$. SCDE of Lie (3.5) has unique analytical solution in the neighborhood of unity element $e \in G$ with initial condition (3.6) if and only if the conditions

$$
A_{\alpha \beta} A_{t s}-A_{t s} A_{\alpha \beta}=\sum_{t=1}^{m} \sum_{s=1}^{m} c_{\beta \beta t s}^{i j} A_{i j},(\alpha, \beta, t, s=1,2, \ldots, m)
$$


are fulfilled. If the condition of absolutely integrability is satisfied, then the solution of the problem (3.4) - (3.5) can be presented in the form

$y(f)=\eta+\sum_{k=1}^{\infty} \frac{1}{k !}\left\{\sum_{i_{1} j_{1} \ldots i_{k} j_{k}}\left(\frac{\partial y(f)}{\partial \alpha_{i_{1} j_{1}}(f) \ldots \partial \alpha_{i_{k} j_{k}}(f)}\right) \prod_{f=c}^{k}\left(\alpha_{i_{t} j_{t}}(f)-\alpha_{i_{t} j_{t}}(e)\right)\right\}$

Where $\sum_{i_{1} j_{1} \ldots i_{k} j_{k}}=\sum_{i_{1}}^{m} \sum_{j_{1}}^{m} \ldots \sum_{i_{k}}^{m} \sum_{j_{k}}^{m} ; c_{\alpha \beta t s}^{i j}$ are the structure real constants of Lie group, satisfying the conditions of antisymmetry and Jakobi identities, and the derivatives of $k$ order of the function $y(f)$ at the point $f=e \in G$ are determined from the following recurrent operator correlations:

$$
\begin{gathered}
A_{i_{1} j_{1} \ldots i_{k} j_{k} i_{k+1} j_{k+1}}=\sum_{t_{1} s_{1} \ldots t_{k} s_{k}}\left\{C_{t_{1} s_{1} i_{k+1} j_{k+1}}^{i_{1} j_{1}} \delta_{t_{2} s_{2}}^{i_{2} j_{2}} \ldots \delta_{t_{k} s_{k}}^{i_{k} j_{k}}+C_{t_{2} s_{2} i_{k+1} j_{k+1}}^{i_{2} s_{2}} \delta_{t_{1} s_{1}}^{i_{1} j_{1}} \delta_{t_{3} s_{3}}^{i_{3} j_{3}} \ldots\right. \\
\left.\delta_{t_{k} s}^{i_{k} j_{k}}+C_{t_{k} s_{k} i_{k+1} j_{k+1}}^{i_{k} j_{k}} \delta_{t_{1} s_{1}}^{i_{1} j_{1}} \ldots \delta_{t_{k-1} s_{k-1}}^{i_{k-1} j_{k-1}}\right\} \times A_{t_{1} s_{1} \ldots t_{k} s_{k}}+A_{i_{1} j_{1} \ldots i_{k} j_{k}} A_{i_{k+1} j_{k+1}}
\end{gathered}
$$

the row (3.7) converges for

$$
\sum_{t=1}^{m} \sum_{s=1}^{m}\left|\alpha_{t s}(f)-\alpha_{t s}(e)\right|<\frac{1}{2 d}(d \geq 1)
$$

\section{REFERENCES}

[1] M. B.Ragimov The beginning of multidimensional spectral analysis of linear operators and its applications. Publish house "Baku University" ,Baku, 2001, 206p.

[2] Shilov G. E. Mathematical Analysis. Finite dimensional linear space, Moscow, "Nauka", 1969, 332p.

[3] N.Bourbaki Groups and Lie algebras, "Mir", Moscow, 1976, 496p.

[4] J.Dedonne Foundations of modern analysis, "Mir", Moscow, 1964

[5] L.Garding Note on continuous representations of Lie groups, Proc. Acad Sci., USA, 33(1947), 331-332p.

[6] M. B.Ragimov Spectral theory of operator- valued maps with commute domain, DAN Azerb. SSR, 1982, v.38, N 4, p.9-12.

[7] M. B.Ragimov To the spectral theory of infinite number of closed commuting between each other linear operators, DAN Azerb. SSR, 1980, v.36, N 2, p.13-17.

[8] M. B.Ragimov On the Taylor spectrum of infinite number of commuting collections of the operators, DAN Azerb. SSR, 1984, v.40, N10 , p.3-5.

[9] M. B.Ragimov Some questions of functional and overdetermined system of differential equations in partial derivatives, I, II, Ucheniye zapiski ASU, ser.phys.-math.n., Baku1977. N4, p.67-75, Baku-1978, N6, p.61-65.

[10] E Hill. F.Phillips Functional analysis and semi-groups M. Li., 1962.

[11] B.L Van Der Varden, Method of Group Theory in Quantum Mechanic DNTVU, Kharkov, 1938,199p.

\section{Received: April 24, 2006}

